\title{
Breathe with Me: A Virtual Meditation Coach
}

\author{
Ameneh Shamekhi, ${ }^{1}$ Timothy Bickmore ${ }^{1}$ \\ ${ }^{1}$ College of Computer and Information Science, Northeastern University, Boston, MA \\ \{ameneh,bickmore\}@ccs.neu.edu
}

\begin{abstract}
A virtual agent that guides users through mindfulness meditation sessions is described. The agent uses input from a respiration sensor to both respond to user breathing rate and use deep breaths as a continuation and acknowledgment signal. A pilot evaluation study comparing the agent to a selfhelp video indicates that users are very receptive to the virtual meditation coach, and that it is more effective at reducing anxiety and increasing mindfulness and flow state compared to the video.
\end{abstract}

\section{Introduction}

Meditation involves a complex set of techniques designed to promote relaxation and emotional balance. It has been found to be therapeutic for a wide range of mental and physical conditions, including chronic pain, depression, anxiety, substance use, and insomnia, and is also an effective buffer against conditions such as post-traumatic stress disorder. Meditation works by increasing meta-cognitive awareness of one's mental states - both cognitive and affective-allowing the practitioner to more effectively cope with extreme cognitions, affective states and moods [6]. The act of meditation thus bridges the divide between cognition and affect in a useful, therapeutic manner.

Meditation takes practice to master, and, unfortunately, most individuals do not have access to classes or experts who can effectively teach them the techniques required for mastery. Many self-help books, videos, and audio programs exist to provide meditation instruction, but they are neither tailored to individual needs nor interactive. In this project, we are developing a virtual agent that plays the role of a meditation coach that is designed to help practitioners focus on their breathing and relax. The meditation agent guides users through an interactive meditation session while monitoring their breathing and providing feedback (Figure 1). 

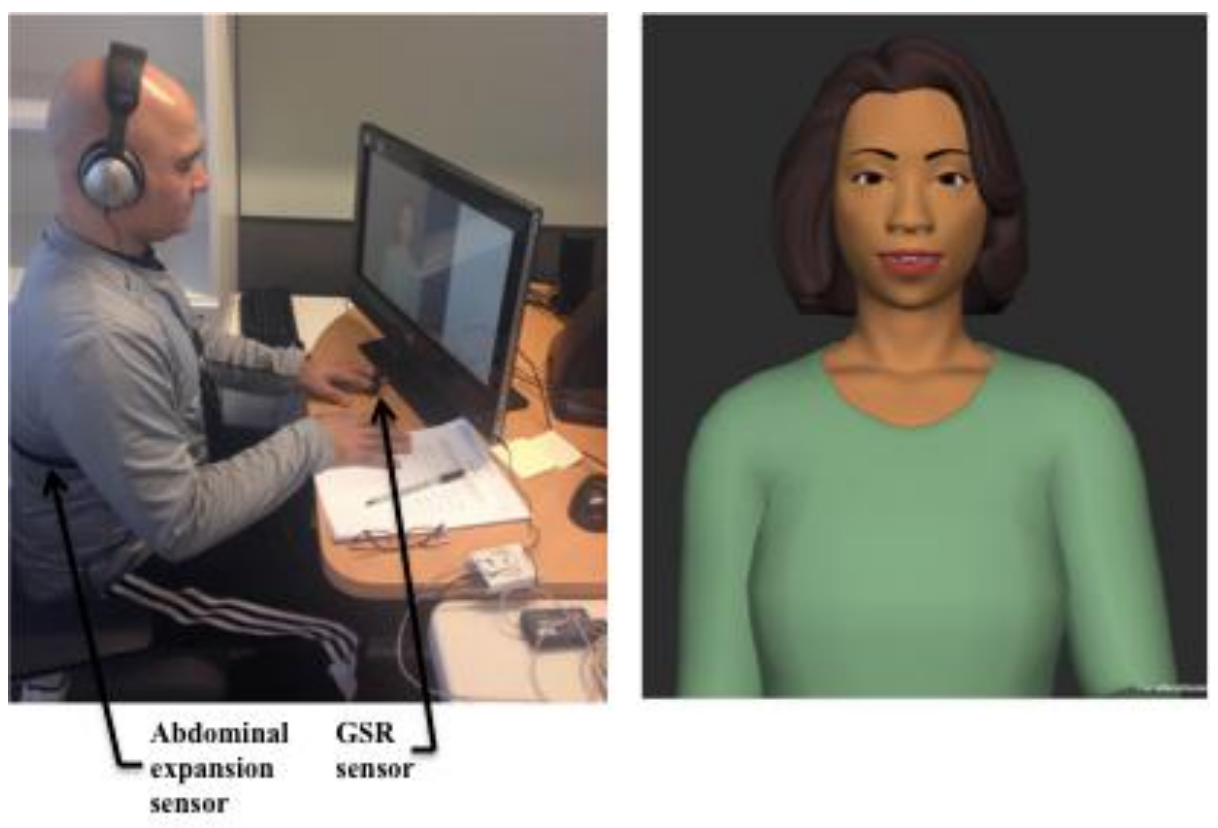

Fig. 1. The Meditation Agent

Experimental setup (left). Closeup of agent (right)

\section{The Virtual Meditation Coach}

The virtual meditation coach is an embodied conversational agent developed to guide novice users through a mindfulness meditation session and help them relax. The agent speaks using synthetic speech driven by a dialogue engine, with synchronized animated co-verbal behavior (Figure 1). In order to evaluate the efficacy of this medium compared to ubiquitous self-help materials without introducing contentrelated confounds, we scripted the agent dialogue to match that from a self-help video as closely as possible. We used a publicly-available video that was approximately 10 minutes in length and featured a mid-shot of an instructor facing the camera throughout the entire session.

To make the virtual meditation coach interactive, we added a breathing sensor to the system. The Respiration Sensor from Thought Technology Ltd. is a flexible strap worn around the chest that measures abdominal expansion (Figure 1, left). Data is processed through a Thought Technology ProComp2 Biofeedback device, and recorded and processed in the agent dialogue system. The dialogue system can respond to either absolute expansion level, or breathing rate. We used the former signal to allow users to signal that they are ready to continue during a meditation session by simply taking a deep breath. We felt that this was a more natural and less disruptive input modality than touch screen input or even speech while a user is meditating. We used breathing rate at certain key points during the meditation to 
ensure users are not breathing too rapidly, and asking them to slow down before continuing if they are.

\section{Pilot Evaluation Study}

We evaluated the virtual meditation coach in a randomized, counterbalanced, withinsubjects experiment, in which we compared it to the self-help video of a human meditation instructor. In order to ensure that study participants were in similar, nonresting, emotional states prior to each meditation session, we induced mild frustration prior to the start of each session. This was accomplished by asking them to solve 10 challenging, timed math problems, in which they were forced to complete their answers very quickly with loud audio feedback regarding the correctness of their answers, with a pleasant tone for correct answers and a grating klaxon for incorrect ones. For the last 5 problems, participants were always given the klaxon regardless of their response.

Participants. Participants were recruited from an online job recruiting site, and were required to be 18 years of age or older, and to speak and read English. Participants were compensated for their time. We recruited 9 participants: $56 \%$ male, aged 21 to 66 (mean 46.1). Most (66\%) had never tried mindfulness meditation.

Measures. We assessed their baseline mindful attention using the Mindful Attention Awareness Scale [1]. Following each meditation session we assessed State Anxiety [2], Positive and Negative Affect (PANAS [3]), Mindfulness (Toronto Mindfulness scale [4]), Flow state [5], and satisfaction with the instructor and experience (Table 1). Physiological arousal was measured using galvanic skin response (GSR) sensors from Thought Technology Ltd, worn on the non-dominant hand for each participant (Figure 1, left). We computed the difference between the first 30 seconds (averaged) and final 30 seconds of each meditation session as our measure for change in arousal during meditation.

Table 1. Satisfaction Questions (mean (sd))

Tests using non-parametric Wilcoxon Signed Ranks

\begin{tabular}{|c|c|c|c|c|c|}
\hline Item & Anchor 1 & Anchor 7 & AGENT & HUMAN & p \\
\hline $\begin{array}{l}\text { How satisfied were you with the } \\
\text { instructor? }\end{array}$ & Not at all & $\begin{array}{c}\text { Very } \\
\text { satisfied }\end{array}$ & $\begin{array}{c}6.11 \\
(1.69)\end{array}$ & $\begin{array}{c}6.56 \\
(0.73)\end{array}$ & .655 \\
\hline $\begin{array}{l}\text { How aware was the instructor of } \\
\text { your breathing? }\end{array}$ & Not at all & $\begin{array}{l}\text { Very } \\
\text { aware }\end{array}$ & $\begin{array}{c}5.22 \\
(2.22) \\
\end{array}$ & $\begin{array}{c}3.89 \\
(2.09) \\
\end{array}$ & .090 \\
\hline $\begin{array}{l}\text { How would you characterize } \\
\text { your relationship with the } \\
\text { instructor? }\end{array}$ & $\begin{array}{l}\text { Complete } \\
\text { stranger }\end{array}$ & $\begin{array}{l}\text { Close } \\
\text { friend }\end{array}$ & $\begin{array}{c}2.88 \\
(2.23)\end{array}$ & $\begin{array}{c}2.67 \\
(2.65)\end{array}$ & 1.00 \\
\hline
\end{tabular}

Procedure. Participants were fitted with the respiration and GSR sensors and randomized to one of the two treatment conditions (AGENT vs. VIDEO). The participant then completed the frustration induction (math test), followed by a calibration procedure for the respiration sensor, followed by the selected meditation session. Participants then completed the post-test questionnaires. Participants were 
then assigned to the other treatment condition (AGENT or VIDEO) and repeated the frustration induction, calibration, meditation, and post-test measurements.

Results. As this is a small pilot study, we set our level of significance at 0.1 . Results are summarized in Tables 1 and 2. Participants reported that the agent was significantly more aware of their breathing compared to the video-based instructor, although there were no significant differences in their overall satisfaction rating. Participants also reported significantly lower levels of anxiety following the session with the agent compared to the video-based human instructor, as well as scoring significantly higher on mindfulness. However, GSR readings indicated that the video led to greater reduction in physiological arousal compared to the agent. There were no significant differences on positive affect (PANAS) or flow, although the agent did score significantly higher on one subscale of flow - Transformation of Time, paired $\mathrm{t}(8)=2.0, \mathrm{p}=.081$ - with no significant differences on the other subscales.

Table 2. Comparative Outcomes for AGENT vs. VIDEO treatments (mean (SD))

Tests using paired-sample t-tests

\begin{tabular}{|l|c|c|c|}
\hline \multicolumn{1}{|c|}{ Measurement } & AGENT & VIDEO & p \\
\hline State Anxiety & $2.92(0.55)$ & $3.19(0.56)$ & .077 \\
\hline Positive Affect & $3.69(0.95)$ & $3.47(0.83)$ & .449 \\
\hline Flow & $3.60(0.71)$ & $3.53(0.64)$ & .384 \\
\hline Mindfulness & $2.72(0.74)$ & $2.55(0.78)$ & .084 \\
\hline Physiological Arousal (post-pre) & $0.07(0.28)$ & $-0.14(0.87)$ & .016 \\
\hline
\end{tabular}

Conclusion and Future Work. We developed an interactive virtual meditation coach that is responsive to user breathing. Participants felt it was effective, liked the interactivity, and felt that breathing was an appropriate input modality for the system. The agent was significantly more effective than a videotaped meditation instructor at reducing anxiety and increasing mindfulness. We are currently extending the system to be more adaptive to users' needs, to be more tailored and interactive during the meditation process. We are investigating meaningful breathing patterns during meditation and dynamic adaptations that can be made to the meditation instruction. We are also planning a series of evaluation studies to test the efficacy of the resulting system.

\section{References}

1. Brown, K. and R. Ryan, The benefits of being present: Mindfulness and its role in psychological well-being. J. Personality and Social Psychology, 2003. 84: p. 822-848.

2. Marteau, T.M. and H. Bekker, The development of a six-item short-form of the state scale of the Spielberger State-Trait Anxiety Inventory (STAI). British Journal of Clinical Psychology, 1992. 31: p. 301-306.

3. Watson, D., L. Clark, and A. Tellegen, Development and validation of brief measures of positive and negative affect: the PANAS scales. J personality and Social Psychology, 1988. 54: p. 1063-1070.

4. Lau, M., et al., The Toronto Mindfulness Scale: Development and Validation. Journal of clinical psychology, 2006. 62(12): p. 1445-1467.

5. Tenenbaum, G., G. Fogarty, and S. Jackson, The flow experience: A Rasch Analysis of Jackson's Flow State Scale. Journal of Outcome Measurement. 3(3): p. 278- 294. 